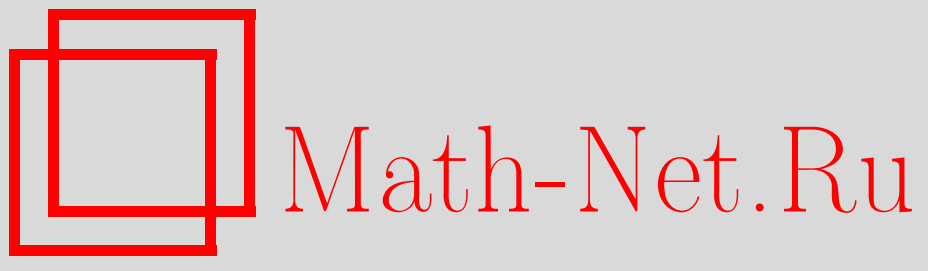

С. А. Гусев, Н. Г. Докучаев, О дифференцировании функционалов, содержащих время первого выхода диффузионного процесса из области, Теория вероятн. и ее примен., 2014, том 59, выпуск 1, 159-168

DOI: https://doi.org/10.4213/tvp4555

Использование Общероссийского математического портала Math-Net.Ru подразумевает, что вы прочитали и согласны с пользовательским соглашением http://www . mathnet.ru/rus/agreement

Параметры загрузки:

IP: 54.224 .60 .19

26 апреля 2023 г., $12: 49: 46$

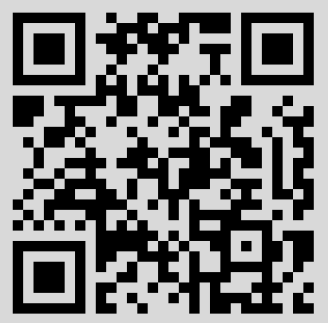


9. Борисов И. С. Об одном критерии марковости гауссовских случайных процессов. - Теория вероятн. и ее примен., 1982, т. 27, в. 4, с. 802-805.

10. Борисов И.С., Быстров А.А. Построение стохастического интеграла от неслучайной функции без условия ортогональности интегрирующей меры. - Теория вероятн. и ее примен., 2005, т. 50, в. 1, с. 52-80.

11. Борисов И. С., Володько Н. В. Экспоненциальные неравенства для распределений $U$ - и $V$ - статистик от зависимых наблюдений. - Матем. труды, 2008, т. 11, в. 2, c. $3-19$.

12. Гихман И. И., Скороход А. В. Теория случайных процессов. Т. 1. М.: Наука, 1971, $664 \mathrm{c}$.

13. Филиппова A. А. Теорема Мизеса о предельном поведении функционалов от эмпирических функций распределения и ее статистические применения. — Теория вероятн. и ее примен., 1962, т. 7, в. 1, с. 26-60.

Поступила в редакцию

1.IX.2012

Исправленный вариант

13.IV.2013

(c) $2014 \Gamma$.

ГУСЕВ С. А.*, ДОКУЧАЕВ Н. Г.**

\title{
О ДИФФЕРЕНЦИРОВАНИИ ФУНКЦИОНАЛОВ, СОДЕРЖАЩИХ ВРЕМЯ ПЕРВОГО ВЫХОДА ДИФФУЗИОННОГО ПРОЦЕССА ИЗ ОБЛАСТИ ${ }^{1)}$
}

\begin{abstract}
Одной из проблем при дифференцировании функционалов диффузионных процессов в областях с поглощающими границами является вычисление производных по параметрам для времени первого выхода случайного процесса из области $\tau$. Ранее в работе [5] был предложен метод решения этой проблемы, но при этом накладывалось ограничительное и трудно проверяемое условие существования среднеквадратических производных $\tau$ по параметрам. В данной работе показано, что при некоторых дополнительных предположениях это условие может быть снято.
\end{abstract}

Ключевые слова и фразы: диффузионный процесс, время первого выхода, поглощающая граница, производные по параметрам.

1. Введение. Необходимость дифференцирования по параметрам функционалов случайных процессов возникает в задачах стохастической оптимизации. Решение таких задач осложняется в случаях, когда функционалы содержат время первого выхода $\tau$ случайного процесса из области. Известно, например, что время первого выхода для гладких процессов не является непрерывным относительно малых изменений параметров. С другой стороны, для случайных диффузионных процессов имеется некоторая регулярность математических ожиданий, содержащих время первого выхода случайного процесса [1], [2]. Однако эта регулярность не гарантирует существование производных $\tau$. Дифференцирование математических ожиданий функционалов, содержащих $\tau$, в принципе возможно (например, через дифференцирование по параметру соответствующих решений краевых задач для уравнений Колмогорова в случае

* Институт вычислительной математики и математической геофизики СО РАН, Новосибирск, Россия; sag@osmf.sscc.ru

** Department of Mathematics and Statistics, Curtin University, Perth, Australia; email: n.dokuchaev@curtin.edu.au

1) Работа поддержана Австралийским исследовательским советом (ARC) (грант DP120100928), научной программой «Ведущие научные школы» (грант НШ5111.2014.1) и РФФИ (грант № 14-01-00340-а). 
диффузионных процессов). Но практическое вычисление этих производных является сложной задачей.

В настоящее время имеется не так много работ, посвященных проблеме дифференцирования по параметрам функционалов, содержащих $\tau$. При этом задачи, в которых требуется определение соответствующих производных, встречаются в различных приложениях. Необходимость вычисления производных по параметрам решений параболических уравнений возникает, например, при использовании градиентных методов оптимизации для решения задач оптимального выбора параметров для этих уравнений. Соответственно, дифференцирование функционалов от случайных процессов, содержащих $\tau$, требуется при решении задач стохастической оптимизации градиентными методами. В частности, определение оценок производных по параметрам функционалов диффузионных процессов встречается в работах по финансовой математике. Например, в [3], [4] оценки производных по параметрам цены опциона определяются на основе стохастического исчисления Маллявэна. В этих работах рассматриваются функционалы несколько иного типа, чем приведенный ниже функционал (2), хотя для опционов барьерного типа задачу можно преобразовать так, что в ней будет фигурировать функционал вида (2).

В [5] было предложено выразить производные от функционалов, содержащих $\tau$, из уравнения, которое получается в результате применения формулы Ито к некоторой функции, обращающейся вместе со своими первыми производными в нуль на границе. При этом накладывалось условие существования среднеквадратических производных $\tau$ по параметрам. В реальности проверка этого условия затруднительна; более того, в настоящее время неизвестны примеры, когда оно выполнено.

В предлагаемой работе показано, что полученная в [5] формула справедлива без этого ограничительного условия, если коэффициенты уравнения являются достаточно гладкими функциями.

2. Постановка задачи. Предположим, что даны связная ограниченная область $G \subset \mathbf{R}^{d}$ с регулярной границей $\partial G$ (см. далее), вероятностное пространство $(\Omega, \mathscr{F}, \mathbf{P})$, для которого задана неубывающая последовательность $\sigma$-алгебр $\mathscr{F}_{t} \subseteq \mathscr{F}, t \geqslant 0$. Пусть задан $W$. - $d$-мерный винеровский процесс, прогрессивно измеримый относительно $\mathscr{F}_{t} ;$ для $s>t$ разность $W_{s}-W_{t}$ независима от $\sigma$-алгебры $\mathscr{F}_{t}$.

Зададим открытое множество $U \subset \mathbf{R}^{m}$. Пусть $x \in G$ и $t \in[0, T)$. Рассмотрим $d$-мерный случайный процесс $X_{s}=X_{s}(\theta)$, который зависит от векторного параметра $\theta \in U$ и описывается следующим векторным СДУ:

$$
X_{s}(\theta)=x+\int_{t}^{s} a\left(v, X_{v}(\theta), \theta\right) d v+\int_{t}^{s} \sigma\left(v, X_{v}(\theta), \theta\right) d W_{v}
$$

с измеримыми функциями $a:[0, \infty) \times \mathbf{R}^{d} \times U \rightarrow \mathbf{R}^{d}$ и $\sigma:[0, \infty) \times \mathbf{R}^{d} \times U \rightarrow \mathbf{R}^{d \times d}$. Мы предполагаем, что коэффициенты СДУ (1) удовлетворяют следующему условию.

А) Функции $a, \sigma$ ограничены и существует константа $\mathscr{K}$ такая, что для всех $\theta \in U, v \geqslant 0, x, y \in \mathbf{R}^{d}, i, j \in\{1, \ldots, d\}$ выполняется неравенство

$$
\left|a_{i}(v, x, \theta)-a_{i}(v, y, \theta)\right|+\left|\sigma_{i j}(v, x, \theta)-\sigma_{i j}(v, y, \theta)\right| \leqslant \mathscr{K}|x-y| .
$$

Этих предположений относительно $a, \sigma$ достаточно [6] для того, чтобы при любом $\theta \in U$ существовал $\mathscr{F}_{s}$-измеримый процесс $X_{s}$ такой, что для него при всех $s \geqslant 0 \mathrm{c}$ вероятностью 1 выполняется равенство (1).

Символом $E_{t, x}$ будем обозначать математическое ожидание относительно вероятностной меры $P_{t, x}$, соответствующей случайному процессу, исходящему в момент времени $t$ из точки $x$ (определение $P_{t, x}$ см., например, в [7, с. 381]).

В приложениях встречаются математические ожидания вида

$$
u(t, x, \theta)=E_{t, x}\left[\varphi\left(X_{T}(\theta), \theta\right) \chi_{\tau>T}+\int_{t}^{T \wedge \tau} f\left(v, X_{v}(\theta), \theta\right) d v\right],
$$


где $\tau=\inf \left\{v: v>t, X_{v} \notin G\right\}-$ время первого выхода процесса $X$. из области, $\chi_{A}-$ индикаторная функция множества $A$.

Обозначим $Q_{T}=(0, T) \times G$. Известно, что при некоторых ограничениях на $\varphi$ и $f$ значение функционала $(2)$ в точке $(t, x) \in Q_{T}$ совпадает с решением следующей краевой задачи для параболического уравнения:

$$
\begin{aligned}
& L u+f(t, x, \theta)=0, \quad t \in(0, T), \quad(t, x) \in Q_{T}, \\
& u(T, x, \theta)=\varphi(x, \theta), \quad x \in G, \\
& u(t, x, \theta)=0, \quad x \in \partial G .
\end{aligned}
$$

Оператор $L=L(\theta)$ в уравнении (2) задан формулой

$$
L \equiv \frac{\partial}{\partial t}+\frac{1}{2} \sum_{i, j=1}^{d} b_{i j}(t, x, \theta) \partial_{x_{i}, x_{j}}^{2}+\sum_{i=1}^{d} a_{i}(t, x, \theta) \partial_{x_{i}},
$$

где $b_{i j}$ - элементы матрицы $B \equiv \sigma \sigma^{*}$.

Приведенный в (2) функционал содержит $\tau$. В данной статье исследуется возможность дифференцирования математических ожиданий вида (2). В связи с этим дополнительно к условиям А) вводятся следующие предположения.

В) Матричная функция $B(t, x, \theta)=\left\{b_{i j}(t, x, \theta)\right\}$ равномерно невырождена:

$$
B(t, x, \theta) \geqslant \alpha_{0} I
$$

для некоторого $\alpha_{0}>0$.

C) Граница гладкая класса не ниже $C^{4}$ и, кроме того, в $[0, \infty) \times \mathbf{R}^{d} \times U$ непрерывны и ограничены следующие производные:

$$
\frac{\partial a}{\partial x}, \frac{\partial^{2} a}{\partial x^{2}}, \frac{\partial a}{\partial \theta_{i}}, \frac{\partial^{2} a}{\partial x \partial \theta_{i}}, \frac{\partial \sigma}{\partial x}, \frac{\partial^{2} \sigma}{\partial x^{2}}, \frac{\partial \sigma}{\partial \theta_{i}}, \frac{\partial^{2} \sigma}{\partial x \partial \theta_{i}}, \frac{\partial a}{\partial t}, \frac{\partial \sigma}{\partial t} .
$$
$Q \times U$.

D) Существуют непрерывные производные $\partial \varphi / \partial x$ и $\partial \varphi / \partial \theta$ при любых $(x, \theta) \in$

Е) Функция $f$ непрерывна на $[0, T] \times \bar{Q}_{T}$ при любом $\theta \in U$ и существуют непрерывные производные $\partial f / \partial x$ и $\partial f / \partial \theta$ при любых $(t, x, \theta) \in Q_{T} \times U$.

$\mathrm{B}$ предположении $\mathrm{E})$ и ниже по тексту черта над обозначением множества означает его замыкание.

Обозначим $G_{\delta}$ подмножество точек $G$, находящихся на расстоянии большем, чем $\delta>0$, от $\partial G$. В [8] даны теоремы существования решений краевых задач для параболических уравнений. При этом тип функционального пространства, к которому принадлежит решение, существенно зависит от исходных данных задачи. Для целей настоящей статьи подойдет любое решение, которое имеет непрерывную классическую производную $\partial u / \partial x$ в точках из любого непустого множества вида $(0, T-\delta) \times G_{\delta}$. Для решения задачи (2)-(5) это условие выполнено в силу следующих теорем из [8, гл. III, теорема $4.2 ;$ гл. IV, теоремы 5.2 и 9.1]. При этом входные данные задачи (2)-(5) должны быть такими, чтобы выполнялись приведенные в этих теоремах достаточные условия существования решения.

3. Основной результат: вычисление $\partial u / \partial \theta$. В дальнейшем, для сокращения записи формул, будем считать, что в уравнении (1) присутствует один скалярный параметр $\theta \in U(U \subset \mathbf{R}-$ некоторый интервал), отметив при этом, что все результаты легко переносятся на случай векторного параметра.

Если формально продифференцировать по $\theta$ функционал (2), то получим

$$
\begin{aligned}
\frac{\partial u}{\partial \theta}(t, x)=E_{t, x} & {\left[\left(\frac{\partial \varphi}{\partial x}\left(T, X_{T}, \theta\right) Z_{T}+\frac{\partial \varphi}{\partial \theta}\left(T, X_{T}, \theta\right)\right) \chi_{\tau>T}\right.} \\
+ & \left.\int_{t}^{T \wedge \tau}\left(\frac{\partial f}{\partial x}\left(v, X_{v}, \theta\right) Z_{v}+\frac{\partial f}{\partial \theta}\left(v, X_{v}, \theta\right)\right) d v\right]+\Phi(\theta),
\end{aligned}
$$


где

$$
\Phi(\theta):=\lim _{\Delta \theta \rightarrow 0} E_{t, x}\left(\frac{\tau(\theta+\Delta \theta)-\tau(\theta)}{\Delta \theta} f\left(\tau, X_{\tau}\right) \chi_{\tau<T}\right)
$$

(если этот предел существует) и процесс

$$
Z_{s}(\theta)=\int_{t}^{s}\left(\frac{\partial a}{\partial x} Z_{v}(\theta)+\frac{\partial a}{\partial \theta}\right) d v+\int_{t}^{s}\left(\frac{\partial \sigma}{\partial x} Z_{v}(\theta)+\frac{\partial \sigma}{\partial \theta}\right) d W(v)
$$

является среднеквадратической производной $\partial X . / \partial \theta$ по параметру решения СДУ $(1)$. Известно (см., например, [9]), что сделанные выше предположения обеспечивают существование среднеквадратической производной $Z_{s}(\theta)=\partial X$. $/ \partial \theta$, которая может быть получена в результате решения системы СДУ (1), (8).

Для определения первого слагаемого в (7) требуется только знать процесс $(X .(\theta), Z .(\theta))$, который является марковским, так как система СДУ $(1),(8)$ удовлетворяет условиям существования сильного решения. Если рассматривать полученную в статье формулу для определения производных вида $\partial u / \partial \theta$ с точки зрения практического применения, то для определения пар вида $(X .(\theta), Z .(\theta))$ можно использовать численные методы [5].

При определении второго слагаемого в (7) возникает необходимость учитывать зависимость $\tau$ от $\theta$, и здесь возникают трудности, о которых говорилось во введении.

Из сказанного следует, что основная проблема в нахождении производных $\partial u / \partial \theta$ с помощью формулы (7) состоит в определении $\Phi(\theta)$. В данной работе, так же как и в [5], мы предлагаем формулу для определения $\Phi(\theta)$, полученную на основе перехода к пределу при $\Delta \theta \rightarrow 0$, в которой не используется $\partial \tau / \partial \theta$.

Для доказательства полученной ниже формулы для $\Phi(\theta)$ мы используем построенную в работе [2] оценку математического ожидания модуля разности значений времени выхода двух диффузионных процессов, описываемых уравнением (1). В [2] для различных коэффициентов $a^{(1)}, \sigma^{(1)}$ и $a^{(2)}, \sigma^{(2)}$ в СДУ (1) при условиях ограниченности этих коэффициентов и их производных по $x$ на бесконечном цилиндре $Q_{\infty} \equiv(0, \infty) \times G$ доказано следующее неравенство [2, теорема 2.3]:

$$
E_{t, x}\left[\frac{1}{\lambda}\left(\exp \left(\lambda\left|\tau_{1}-\tau_{2}\right|\right)-1\right)\right] \leqslant \max _{k=1,2} \sup _{(t, x) \in Q_{\infty}}\left|\frac{d v_{k}}{d x}\right| E_{t, x}\left|X_{\tau_{1} \wedge \tau_{2}}^{(1)}-X_{\tau_{1} \wedge \tau_{2}}^{(2)}\right|,
$$

где $\lambda>0$ - константа, зависящая от $d, G, \sup _{Q_{\infty}} a^{(k)}(k=1,2), \alpha_{0}$. В контексте данной статьи коэффициенты $a^{(k)}, \sigma^{(k)}(k=1,2)$ суть коэффициенты СДУ (1), взятые при различных значениях параметра $\theta=\theta_{k}$, т.е. $a^{(k)}(t, x) \equiv a\left(t, x, \theta_{k}\right)$, $\sigma^{(k)}(t, x) \equiv \sigma\left(t, x, \theta_{k}\right)$. В (9) использованы следующие обозначения: $X^{(k)}-$ случайный процесс, который получается, если в СДУ (1) подставить вместо $a, \sigma$ соответственно коэффициенты $a^{(k)}, \sigma^{(k)}(k=1,2) ; \tau_{k}$ - время первого выхода $X^{(k)}$ из области $G$; $v_{k}$ - решение следующей краевой задачи в $Q_{\infty}$ :

$$
\begin{aligned}
& L^{(k)} v_{k}+\lambda v_{k}+1=0, \\
& \left.v_{k}(t, x)\right|_{x \in \partial G}=0, \\
& \underset{t>0}{\operatorname{ess} \sup }\left\|v_{k}(t, \cdot)\right\|_{L_{2}(G)}<\infty,
\end{aligned}
$$

где $L^{(k)}$ - параболический оператор вида $(6)$, у которого коэффициентами при первых и вторых производных по $x$ являются соответственно элементы вектора $a^{(k)}$ и матрицы $B^{(k)} \equiv \sigma^{(k)}\left(\sigma^{(k)}\right)^{*}$. По теореме 2.1 в [2] $\sup _{x, t, \theta_{k}}\left|\partial v_{k} / \partial x\right|<+\infty$.

Для выражения под знаком математического ожидания в левой части (9) выполняется очевидное неравенство

$$
\left|\tau_{1}-\tau_{2}\right|^{p} \leqslant p ! \lambda^{-p}\left(\exp \left(\lambda\left|\tau_{1}-\tau_{2}\right|\right)-1\right) \quad \text { для } p=1,2, \ldots
$$


Неравенства (9), (13) мы используем ниже, взяв в качестве $X^{(1)}, X^{(2)}$ процессы $X .(\theta+\Delta \theta), \quad X .(\theta)$ и заменив в (9) $\max _{k=1,2} \sup _{(t, x) \in Q_{\infty}}\left|d v_{k} / d x\right|$ на $\sup _{(t, x, \theta) \in Q_{\infty} \times U}|d v / d x|$, где $v$ - решение задачи вида (10)-(12) с оператором (6).

Лемма 1. Для любого иелого $p \geqslant 1$ имеет место сходимость

$$
E_{t, x}|\tau(\theta+\Delta \theta)-\tau(\theta)|^{p} \rightarrow 0 \quad \text { nрu } \Delta \theta \rightarrow 0 .
$$

Д о к а з а т е л ь с т в о. Заметим, что решение СДУ (1) непрерывно в среднем квадратическом по $\theta$. Обозначим $\widetilde{\tau}(\theta, \Delta \theta)=\tau(\theta) \wedge \tau(\theta+\Delta \theta)$. Применяя (9) и (13) к $X .(\theta+\Delta \theta)$ и $X .(\theta)$, получим оценку математического ожидания для $|\tau(\theta+\Delta \theta)-\tau(\theta)|^{p}$ :

$$
E_{t, x}|\tau(\theta+\Delta \theta)-\tau(\theta)|^{p} \leqslant C(p) E_{t, x}\left|X_{\tilde{\tau}(\theta, \Delta \theta)}(\theta+\Delta \theta)-X_{\tilde{\tau}(\theta, \Delta \theta)}(\theta)\right|,
$$

где $C(p)=p ! \lambda^{-p} \sup _{(t, x, \theta) \in Q_{\infty} \times U}|d v / d x|$.

Случайный процесс $X .(\theta)$ непрерывен по $\theta$ в среднеквадратическом смысле, откуда следует, что $E_{t, x}\left|X_{\tilde{\tau}(\theta, \Delta \theta)}(\theta+\Delta \theta)-X_{\tilde{\tau}(\theta, \Delta \theta)}(\theta)\right| \rightarrow 0$ при $\Delta \theta \rightarrow 0$. Лемма доказана.

Лемма 2. Существует постоянная $K>0$ такая, что при $\Delta \theta \rightarrow 0$

$$
E_{t, x}\left|\frac{\tau(\theta+\Delta \theta)-\tau(\theta)}{\Delta \theta}\right|<K .
$$

Д о к а з а т е л ь с т в о. Мы знаем, что решение СДУ (1) дифференцируемо в среднем квадратическом по $\theta$. Поделим неравенство (15) при $p=1$ на $\Delta \theta$ :

$$
E_{t, x}\left|\frac{\tau(\theta+\Delta \theta)-\tau(\theta)}{\Delta \theta}\right| \leqslant C(1) E_{t, x}\left|\frac{\Delta X_{\tilde{\tau}(\theta, \Delta \theta)}(\theta)}{\Delta \theta}\right|,
$$

где $\Delta X_{\tilde{\tau}(\theta, \Delta \theta)}(\theta)=X_{\tilde{\tau}(\theta, \Delta \theta)}(\theta+\Delta \theta)-X_{\tilde{\tau}(\theta, \Delta \theta)}(\theta)$. Далее, применяя неравенство КошиБуняковского и среднеквадратическую дифференцируемость $X$. по $\theta$, получаем при $\Delta \theta \rightarrow 0$

$$
\begin{aligned}
E_{t, x}\left|\frac{\Delta X_{\tilde{\tau}(\theta, \Delta \theta)}(\theta)}{\Delta \theta}\right| & \leqslant\left[E_{t, x}\left(\frac{\Delta X_{\tilde{\tau}(\theta, \Delta \theta)}(\theta)}{\Delta \theta}\right)^{2}\right]^{1 / 2} \\
& \leqslant \sup _{0 \leqslant v \leqslant T}\left[E_{t, x} \chi_{v \leqslant \tau \wedge T} Z_{v}^{2}(\theta)\right]^{1 / 2},
\end{aligned}
$$

где $Z$. - среднеквадратическая производная $X$. по $\theta$. Величина $E_{t, x} Z_{v}^{2}(\theta)$ ограничена, так как для уравнения (8) выполняются условия теоремы 4 из $[9$, с. 48]. Лемма доказана.

Для произвольной функции $r(x, \theta)$ такой, что $r \in C^{1}\left(\mathbf{R}^{d+1} \rightarrow \mathbf{R}\right)$, будем использовать обозначение

$$
\frac{d}{d \theta} r(X .(\theta), \theta)=\frac{\partial r}{\partial x} \frac{\partial X .(\theta)}{\partial \theta}+\frac{\partial r}{\partial \theta}
$$

где $\partial X .(\theta) / \partial \theta$ - среднеквадратическая производная.

Нижеследующая теорема содержит основной результат статьи.

Теорема 1. Пусть коэффициенты уравнения (1) и функиии $\varphi, f$ удовлетворяют условиям А)-Е). Тогда имеет место формула (9). При этом предел $\Phi(\theta)$ существует и может быть определен с помощью следующей формуль:

$$
\begin{aligned}
\Phi(\theta)=-E_{t, x}\left[\chi _ { \tau < T } \frac { f ( \tau , X _ { \tau } ) } { ( L g ) _ { \tau } } \left(\int_{t}^{\tau}\right.\right. & \frac{d}{d \theta}(L g)_{v} d v \\
& \left.\left.+\int_{t}^{\tau} \frac{d}{d \theta} \sum_{l, j}\left(\frac{\partial g}{\partial x_{l}} \sigma_{l j}\right)_{v} d W_{j}(v)\right)\right],
\end{aligned}
$$

где в качестве g можно выбрать любую функиию класса $C^{4}\left(\mathbf{R}^{d} \rightarrow \mathbf{R}\right)$, которая обращается вместе со своими первыми производными в нуль на $\partial G$, но при этом значения $L g$ не обращаются в нуль на $\partial G$ ни для каких $\theta \in U$. 
3 а м е ч а н и е. Для любой ограниченной области $G$ в пространстве $\mathbf{R}^{d}$ с границей $\partial G$ класса $C^{l}, l \geqslant 2$, можно построить функцию $g(x)$ класса $C^{l}$, которая равна нулю на $\partial G$ вместе со своими первыми производными, и при этом ее вторые производные на границе не обращаются в нуль. Такая функция получается, например, в результате возведения в некоторую целую положительную степень функции расстояния до границы $\partial G$, которая определяется в окрестности $\partial G$ следующим образом $\rho(x)=\min _{y \in \partial G}|x-y|$. Обычно функцию $\rho(x)$ задают так, чтобы она была положительной для точек внутри области и отрицательной для точек, находящихся вне области. Функцию $\rho(x)$ можно продолжить с сохранением ее глацкости на все пространство $\mathbf{R}^{d}$. Построение функций такого типа описано в книге [10]. Следовательно, функция $g$ с требуемыми свойствами всегда существует в предположении, что граница области $C^{4}$-гладкая.

Д о к а з а т ел ь с т в о т е о ре м ы 4 . Для $\psi \in C^{1}\left(\mathbf{R} \times \mathbf{R}^{d} \rightarrow \mathbf{R}\right)$ введем обозначение

$$
R_{j}^{\psi}\left(v, X_{v}, \theta\right)=\sum_{l} \frac{\partial \psi\left(X_{v}(\theta)\right)}{\partial x_{l}} \sigma_{l j}\left(v, X_{v}(\theta), \theta\right) .
$$

Так как функция $g$ равна нулю на границе, то в результате применения формулы Ито получим равенство

$$
0=g(x)+\int_{t}^{\tau(\theta)} L g\left(v, X_{v}(\theta), \theta\right) d v+\int_{t}^{\tau(\theta)} \sum_{j} R_{j}^{g}\left(v, X_{v}(\theta), \theta\right) d W_{j}(v) .
$$

Как и в доказательстве леммы 1 , используем обозначение $\widetilde{\tau}(\theta, \Delta \theta)=\tau(\theta) \wedge \tau(\theta+$ $\Delta \theta)$. Применяя (20) для значений параметра $\theta$ и $\theta+\Delta \theta$, можем записать равенство

$$
\begin{aligned}
0= & \frac{1}{\Delta \theta}\left[\int_{t}^{\tilde{\tau}(\theta, \Delta \theta)}\left(L g\left(v, X_{v}(\theta+\Delta \theta), \theta+\Delta \theta\right)-L g\left(v, X_{v}(\theta), \theta\right)\right) d v\right. \\
& +\int_{\tilde{\tau}(\theta, \Delta \theta)}^{\tau(\theta+\Delta \theta)} L g\left(v, X_{v}(\theta+\Delta \theta), \theta+\Delta \theta\right) d v-\int_{\tilde{\tau}(\theta, \Delta \theta)}^{\tau(\theta)} L g\left(v, X_{v}(\theta), \theta\right) d v \\
& +\int_{t}^{\tilde{\tau}(\theta, \Delta \theta)} \sum_{j}\left(R_{j}^{g}\left(v, X_{v}(\theta+\Delta \theta), \theta+\Delta \theta\right)-R_{j}^{g}\left(v, X_{v}(\theta), \theta\right)\right) d W_{j}(v) \\
& +\int_{\tilde{\tau}(\theta, \Delta \theta)}^{\tau(\theta+\Delta \theta)} \sum_{j} R_{j}^{g}\left(v, X_{v}(\theta+\Delta \theta), \theta+\Delta \theta\right) d W_{j}(v) \\
& \left.-\int_{\tilde{\tau}(\theta, \Delta \theta)}^{\tau(\theta)} \sum_{j} R_{j}^{g}\left(v, X_{v}(\theta), \theta\right) d W_{j}(v)\right] .
\end{aligned}
$$

Умножим (21) на $\chi_{\tau<T} f\left(\tau, X_{\tau}\right) /(L g)_{\tau}$, где $\tau$ берется при значении параметра $\theta$, и рассмотрим предел при $\Delta \theta \rightarrow 0$ математического ожидания правой части полученного равенства.

Покажем, что

$$
\begin{aligned}
\lim _{\Delta \theta \rightarrow 0} E_{t, x}[ & \chi_{\tau(\theta)<T} \frac{f\left(\tau, X_{\tau}\right)}{(L g)_{\tau}} \\
& \left.\times \int_{t}^{\tilde{\tau}(\theta, \Delta \theta)} \frac{L g\left(v, X_{v}(\theta+\Delta \theta), \theta+\Delta \theta\right)-L g\left(v, X_{v}(\theta), \theta\right)}{\Delta \theta} d v\right] \\
=E_{t, x} & {\left[\chi_{\tau(\theta)<T} \frac{f\left(\tau, X_{\tau}\right)}{(L g)_{\tau}} \int_{t}^{\tau(\theta)} \frac{d}{d \theta} L g\left(v, X_{v}(\theta), \theta\right) d v\right] . }
\end{aligned}
$$


Для этого запишем следующее неравенство, которое следует из того, что случайная величина $\chi_{\tau(\theta)<T} \frac{f\left(\tau, X_{\tau}\right)}{(L g)_{\tau}}$ почти всюду ограничена:

$$
\begin{aligned}
& \mid E_{t, x}\left[\chi_{\tau(\theta)<T} \frac{f\left(\tau, X_{\tau}\right)}{(L g)_{\tau}}\right. \\
& \quad \times\left(\int_{t}^{\tilde{\tau}(\theta, \Delta \theta)} \frac{L g\left(v, X_{v}(\theta+\Delta \theta), \theta+\Delta \theta\right)-L g\left(v, X_{v}(\theta), \theta\right)}{\Delta \theta} d v\right. \\
& \left.\left.\quad-\int_{t}^{\tau(\theta)} \frac{d}{d \theta} L g\left(v, X_{v}(\theta), \theta\right) d v\right)\right] \mid \\
& \leqslant C \int_{t}^{T}\left|E_{t, x}\left[\chi_{v<\tilde{\tau}(\theta, \Delta \theta)}\left(\frac{L g\left(v, X_{v}(\theta+\Delta \theta), \theta+\Delta \theta\right)-L g\left(v, X_{v}(\theta), \theta\right)}{\Delta \theta}-\frac{d}{d \theta} L g\left(v, X_{v}(\theta), \theta\right)\right)\right]\right| d v \\
& +C\left|E_{t, x}\left[\int_{\tilde{\tau}(\theta, \Delta \theta)}^{\tau(\theta)} \frac{d}{d \theta} \operatorname{Lg}\left(v, X_{v}(\theta), \theta\right) d v\right]\right|,
\end{aligned}
$$

где $C=\sup _{(v, x, \theta) \in[0, T] \times \partial G \times U}|f(v, x, \theta) /(L g)(v, x, \theta)|$.

Поскольку производные $L g$ по $x, \theta$ непрерывны и ограничены в $\bar{G}$, то можно показать, что при $\Delta \theta \rightarrow 0$

$$
\frac{L g\left(v, X_{v}(\theta+\Delta \theta), \theta+\Delta \theta\right)-L g\left(v, X_{v}(\theta), \theta\right)}{\Delta \theta} \rightarrow \frac{d}{d \theta} L g\left(v, X_{v}(\theta), \theta\right)
$$

по вероятности. Поэтому первое слагаемое в правой части (23) стремится к нулю при $\Delta \theta \rightarrow 0$.

Кроме того, из непрерывности и ограниченности в $\bar{G}$ производных $L g$ следует, что величина $(d / d \theta) L g\left(v, X_{v}(\theta), \theta\right)$ ограничена. Поэтому второе слагаемое в правой части (23) стремится к нулю при $\Delta \theta \rightarrow 0$ на основании леммы 1 :

$$
\begin{gathered}
\left|E_{t, x}\left[\int_{\tilde{\tau}(\theta, \Delta \theta)}^{\tau(\theta)} \frac{d}{d \theta} L g\left(v, X_{v}(\theta), \theta\right) d v\right]\right| \leqslant C_{1} E_{t, x}|\tau(\theta)-\widetilde{\tau}(\theta, \Delta \theta)| \\
\leqslant C_{1} E_{t, x}|\Delta \tau| \rightarrow 0 \quad \text { при } \Delta \theta \rightarrow 0,
\end{gathered}
$$

где

$$
C_{1}=\sup _{(v, x, \theta) \in[0, T] \times \partial G \times U}\left|\frac{d}{d \theta} \operatorname{Lg}\left(v, X_{v}(\theta), \theta\right)\right|, \quad \Delta \tau=\tau(\theta+\Delta \theta)-\tau(\theta) .
$$

Докажем, что при $\Delta \theta \rightarrow 0$

$$
\begin{gathered}
E_{t, x}\left[\chi _ { \tau ( \theta ) < T } \frac { f ( \tau , X _ { \tau } ) } { \Delta \theta ( L g ) _ { \tau } } \left(\int_{\tilde{\tau}(\theta, \Delta \theta)}^{\tau(\theta+\Delta \theta)} \operatorname{Lg}\left(v, X_{v}(\theta+\Delta \theta), \theta+\Delta \theta\right) d v\right.\right. \\
\left.\left.-\int_{\tilde{\tau}(\theta, \Delta \theta)}^{\tau(\theta)} \operatorname{Lg}\left(v, X_{v}(\theta), \theta\right) d v\right)\right] \\
-E_{t, x}\left[\chi_{\tau(\theta)<T} f\left(\tau, X_{\tau}\right) \frac{\Delta \tau}{\Delta \theta}\right] \rightarrow 0 .
\end{gathered}
$$

По построению для интегралов в (25) выполняется равенство

$$
\int_{\tilde{\tau}(\theta, \Delta \theta)}^{\tau(\theta+\Delta \theta)} \operatorname{Lg}\left(v, X_{v}(\theta+\Delta \theta), \theta+\Delta \theta\right) d v-\int_{\tilde{\tau}(\theta, \Delta \theta)}^{\tau(\theta)} L g\left(v, X_{v}(\theta), \theta\right) d v
$$




$$
=\int_{\tau(\theta)}^{\tau(\theta+\Delta \theta)} L g\left(v, X_{v}(\theta+\eta \Delta \theta), \theta+\eta \Delta \theta\right) d v
$$

где

$$
\eta=\left\{\begin{array}{l}
1, \text { если } \tau(\theta)<\tau(\theta+\Delta \theta), \\
0, \text { если } \tau(\theta+\Delta \theta)<\tau(\theta) .
\end{array}\right.
$$

Обозначим $C_{2}=\sup _{(v, x, \theta) \in[0, T] \times \partial G \times U}\left|f(v, x, \theta) /(L g)_{\tau}\right|$. Применяя формулу Ито к $L g\left(v, X_{v}(\theta+\eta \Delta \theta), \theta+\eta \Delta \theta\right)$, с учетом (26) запишем неравенство для модуля выражения в (25):

$$
\begin{gathered}
\left|E_{t, x}\left[\chi_{\tau(\theta)<T} \frac{f\left(\tau, X_{\tau}\right)}{\Delta \theta(L g)_{\tau}} \int_{\tau(\theta)}^{\tau(\theta+\Delta \theta)}\left(L g\left(v, X_{v}(\theta+\eta \Delta \theta), \theta+\eta \Delta \theta\right)-(L g)_{\tau}\right) d v\right]\right| \\
\leqslant C_{2} E_{t, x}\left[\chi_{(\tau(\theta)<T) \&(\eta=1)} \frac{\Delta \tau}{\Delta \theta}\left|L g\left(\tau, X_{\tau}(\theta+\Delta \theta), \theta+\Delta \theta\right)-L g\left(\tau, X_{\tau}(\theta), \theta\right)\right|\right] \\
+C_{2}\left|E_{t, x}\left[\chi_{\tau(\theta)<T} \frac{1}{\Delta \theta} \int_{\tau(\theta)}^{\tau(\theta+\Delta \theta)} d v \int_{\tau(\theta)}^{v} L^{2} g\left(s, X_{s}(\theta+\eta \Delta \theta), \theta+\eta \Delta \theta\right) d s\right]\right| \\
+C_{2} \mid E_{t, x}\left[\chi_{\tau(\theta)<T} \frac{1}{\Delta \theta} \int_{\tau(\theta)}^{\tau(\theta+\Delta \theta)} d v\right. \\
\left.\times \int_{\tau(\theta)}^{v} \sum_{j} R_{j}^{L g}\left(s, X_{s}(\theta+\eta \Delta \theta), \theta+\eta \Delta \theta\right) d W_{j}(s)\right] \mid
\end{gathered}
$$

Очевидно, что второе и третье слагаемые в правой части (27) стремятся к нулю при $\Delta \theta \rightarrow 0$. Рассмотрим $E_{t, x}\left[\chi_{(\tau(\theta)<T) \&(\eta=1)}(\Delta \tau / \Delta \theta)|\Delta L g|\right]$, где $\Delta L g:=L g\left(\tau, X_{\tau}(\theta+\right.$ $\Delta \theta), \theta+\Delta \theta)-L g\left(\tau, X_{\tau}(\theta), \theta\right)$. Как было указано выше, $\Delta L g \rightarrow 0$ при $\Delta \theta \rightarrow 0$ по вероятности. Зададим $\varepsilon_{1}>0, \varepsilon_{2}>0$. Пусть $\delta>0$ таково, что $\mathbf{P}\left\{|\Delta L g|>\varepsilon_{1}\right\}<\varepsilon_{2}$ при $\Delta \theta<\delta$. Тогда при $\Delta \theta<\delta$

$$
E_{t, x}\left[\chi_{(\tau(\theta)<T) \&(\eta=1)} \frac{\Delta \tau}{\Delta \theta}|\Delta L g|\right]<2 K M \varepsilon_{2}+K \varepsilon_{1},
$$

где $K-$ постоянная в неравенстве (16), $M=\sup _{[0, T] \times \partial G \times U} L g$. Таким образом, $(25)$ доказано.

Рассмотрим теперь интегралы по винеровскому процессу в (21). Покажем, что

$$
\begin{aligned}
\lim _{\Delta \theta \rightarrow 0} E_{t, x}[ & \chi_{\tau(\theta)<T} \frac{f\left(\tau, X_{\tau}\right)}{(L g)_{\tau}} \\
& \left.\times \int_{t}^{\tilde{\tau}(\theta, \Delta \theta)} \sum_{j} \frac{R_{j}^{g}\left(v, X_{v}(\theta+\Delta \theta), \theta+\Delta \theta\right)-R_{j}^{g}\left(v, X_{v}(\theta), \theta\right)}{\Delta \theta} d W_{j}(v)\right] \\
=E_{t, x} & {\left[\chi_{\tau(\theta)<T} \frac{f\left(\tau, X_{\tau}\right)}{(L g)_{\tau}} \int_{t}^{\tau(\theta)} \sum_{j} \frac{d}{d \theta}\left(R_{j}^{g}\left(v, X_{v}(\theta), \theta\right)\right) d W_{j}(v)\right] . }
\end{aligned}
$$

Для этого рассмотрим цепочку неравенств

$$
\begin{aligned}
\mid E_{t, x}\left[\chi_{\tau(\theta)<T} \frac{f\left(\tau, X_{\tau}\right)}{(L g)_{\tau}}\right. & \\
\times\left(\int _ { t } ^ { T } \chi _ { v \leqslant \tilde { \tau } ( \theta , \Delta \theta ) } \sum _ { j } \left(\frac{R_{j}^{g}\left(v, X_{v}(\theta+\Delta \theta), \theta+\Delta \theta\right)-R_{j}^{g}\left(v, X_{v}(\theta), \theta\right)}{\Delta \theta}\right.\right. & \left.\left.-\frac{d}{d \theta} R_{j}^{g}\left(v, X_{v}(\theta), \theta\right)\right) d W_{j}(v)\right)
\end{aligned}
$$




$$
\begin{gathered}
\left.-\int_{\tilde{\tau}(\theta, \Delta \theta)}^{\tau(\theta)} \sum_{j} \frac{d}{d \theta}\left(R_{j}^{g}\left(v, X_{v}(\theta), \theta\right)\right) d W_{j}(v)\right] \mid \\
\leqslant C_{2} \int_{t}^{T}\left(\sum _ { j } E _ { t , x } \left(\frac{R_{j}^{g}\left(v, X_{v}(\theta+\Delta \theta), \theta+\Delta \theta\right)-R_{j}^{g}\left(v, X_{v}(\theta), \theta\right)}{\Delta \theta}\right.\right. \\
\left.\left.-\frac{d}{d \theta} R_{j}^{g}\left(v, X_{v}(\theta), \theta\right)\right)^{2}\right)^{1 / 2} d v \\
+C_{2} E_{t, x}\left|\int_{\tilde{\tau}(\theta, \Delta \theta)}^{\tau(\theta)} \sum_{j} \frac{d}{d \theta}\left(R_{j}^{g}\left(v, X_{v}(\theta), \theta\right)\right) d W_{j}(v)\right| .
\end{gathered}
$$

Функция $R_{j}^{g}\left(v, X_{v}(\theta+\Delta \theta), \theta+\Delta \theta\right)$ и ее производные по $x, \theta$ ограничены в $\bar{G}$. Поэтому можно показать, что при $\Delta \theta \rightarrow 0$

$$
\frac{R_{j}^{g}\left(v, X_{v}(\theta+\Delta \theta), \theta+\Delta \theta\right)-R_{j}^{g}\left(v, X_{v}(\theta), \theta\right)}{\Delta \theta} \rightarrow \frac{d}{d \theta} R_{j}^{g}\left(v, X_{v}(\theta), \theta\right)
$$

по вероятности. Отсюда следует, что первое слагаемое в правой части (29) стремится к нулю при $\Delta \theta \rightarrow 0$. Так как функция $(d / d \theta) R_{j}^{g}(\cdot, X ., \theta)$ ограничена, то очевидно, что второе слагаемое также стремится к нулю при $\Delta \theta \rightarrow 0$.

Для завершения доказательства теоремы достаточно показать, что

$$
\begin{aligned}
\lim _{\Delta \theta \rightarrow 0} \frac{1}{\Delta \theta} E_{t, x} & {\left[\int_{\tilde{\tau}(\theta, \Delta \theta)}^{\tau(\theta+\Delta \theta)} \sum_{j} R_{j}^{g}\left(v, X_{v}(\theta+\Delta \theta), \theta+\Delta \theta\right) d W_{j}(v)\right.} \\
- & \left.\int_{\tilde{\tau}(\theta, \Delta \theta)}^{\tau(\theta)} \sum_{j} R_{j}^{g}\left(v, X_{v}(\theta), \theta\right) d W_{j}(v)\right]=0 .
\end{aligned}
$$

Доказательство (30) следует из того, что функция $R_{j}^{g}$ равна нулю на границах отрезков $[\widetilde{\tau}, \tau(\theta)]$ и $[\widetilde{\tau}, \tau(\theta+\Delta \theta)]$. Применяя формулу Ито к этой функции в подынтегральных выражениях в (30), легко убедиться в справедливости (30). Теорема доказана.

Следствие 1. Теорема 1 позволяет получить формулу для $\partial u / \partial \theta$, в которой отсутствует производная $\partial \tau / \partial \theta$ :

$$
\begin{aligned}
\frac{\partial u}{\partial \theta}(t, x)=E_{t, x}[( & \left.\frac{\partial \varphi}{\partial x}\left(X_{T}, \theta\right) Z_{T}+\frac{\partial \varphi}{\partial \theta}\left(X_{T}, \theta\right)\right) \chi_{\tau>T} \\
& +\int_{t}^{T \wedge \tau}\left(\frac{\partial f}{\partial x}\left(v, X_{v}\right) Z_{v}+\frac{\partial f}{\partial \theta}\left(v, X_{v}\right)\right) d v \\
& -\chi_{\tau<T} \frac{f\left(\tau, X_{\tau}\right)}{(L g)_{\tau}}\left(\int_{t}^{\tau} \frac{d}{d \theta}(L g)_{v} d v\right. \\
& \left.\left.+\int_{t}^{\tau} \frac{d}{d \theta} \sum_{l, j}\left(\frac{\partial g}{\partial x_{l}} \sigma_{l j}\right)_{v} d W_{j}(v)\right)\right] .
\end{aligned}
$$

\section{СПИСОК ЛИТЕРАТУРЫ}

1. Докучаев Н.Г. О моментах первого выхода для однородных диффузионных процессов. - Теория вероятн. и ее примен., 1986, т. 31 , в. 3, с. 565-566.

2. Dokuchaev N. Estimates for distances between first exit times via parabolic equations in unbounded cylinders. - Probab. Theory Related Fields, 2004, v. 129, № 2, p. 290314. 
3. Fournié E., Lasry J. M., Lebuchoux J., Lions P. L., Touzi N. Applications of Malliavin calculus to Monte Carlo methods in finance. — Finance Stoch., 1999, v. 3, № 4, p. 391412.

4. Montero M., Kohatsu-Higa A. Malliavin calculus applied to finance. - Phys. A, 2003, v. 320, № $1-4$, p. 548-570.

5. Гусев С.А. Оценка производных по параметрам функционалов диффузионного процесса, движущегося в области с поглощающей границей. - Сиб. журн. вычисл. матем., 2008, т. 11, № 4, с. 385-404.

6. Itô K. On a stochastic integral equation. - Proc. Japan Acad., 1946, v. 22, № 1-4, p. $32-35$.

7. Гихман И. И., Скороход А. В. Введение в теорию случайных процессов. М.: Наука, $1977,567 \mathrm{c.}$

8. Ладыженская О.А., Солонников В.А., Уральчева Н. Н. Линейные и квазилинейные уравнения параболического типа. М.: Наука, 1967, 736 с.

9. Гихман И.И., Скороход А.В. Стохастические дифференциальные уравнения. Киев: Наукова думка, 1968, 354 с.

10. Lieberman G. E. Second Order Parabolic Differential Equations. World Scientific Publ. Co., 2005.

Поступила в редакцию 12.III. 2012

Исправленный вариант

11.XII.2013

(c) 2014 г.

КУЗНЕЦОВА А. А.*, ХОЛЕВО А.С.**

\section{ТЕОРЕМЫ КОДИРОВАНИЯ ДЛЯ ГИБРИДНЫХ КАНАЛОВ. ІІ ${ }^{1)}$}

Настоящая работа продолжает исследование пропускных способностей измерительных (квантово-классических) каналов в наиболее общей постановке, начатое в [10]. В ней дается доказательство теорем кодирования для классической пропускной способности и классической пропускной способности с использованием сцепленности в случае измерительного канала с произвольным выходным алфавитом без предположения, что канал задается ограниченной операторной плотностью.

Ключевые слова и фразы: измерительный канал, гибридная вероятностная система, классическая пропускная способность, сцепленное состояние, теорема кодирования.

1. Введение. Настоящая работа продолжает исследование пропускных способностей измерительных каналов в наиболее общей постановке, начатое в [10]. В ней дается доказательство теорем кодирования для классической пропускной способности (теорема 1) и классической пропускной способности с использованием сцепленности (теорема 2) в случае измерительного канала с произвольным выходным алфавитом при минимальных предположениях регулярности. Утверждение теоремы 2 было ранее доказано в [10] при дополнительном предположении, что измерительный канал задается ограниченной операторной плотностью. В настоящей работе мы освобождаемся от этого ограничения, привлекая обобщение теоремы Радона-Никодима для

* МГТУ им. Н.Э. Баумана, Москва, Россия; e-mail: kuznetsova.a.a@bk.ru

** Математический институт им. В.А.Стеклова РАН, Москва, Россия; е-таil: holevo@mi.ras.ru

1) Работа выполнена при поддержке РФФИ (грант № 12-01-00319) и Российского Квантового Центра. 